\title{
Paediatric renal transplantation: moving forward in the field
}

\author{
Nizam Mamode ${ }^{1,2} \cdot$ Stephen D Marks $^{3}$ (D)
}

Received: 3 July 2017 / Accepted: 12 July 2017 / Published online: 28 July 2017

(C) IPNA 2017

Keywords Renal transplantation · Children · Anatomy · Physiology $\cdot$ Pathophysiology $\cdot$ Immunology

The field of renal transplantation is becoming more complicated, with both clinicians and academics needing to know the latest advances. Although we learn from adult practice how to treat children, children are not small adults. There are inherent differences in anatomy, physiology, pathophysiology, and immunology between infants, children, and adolescents regarding their developing immune systems, making patient management complex for both physicians and surgeons. As children may require multiple renal transplants, subsequent sensitization is a key issue. Although close HLA matching is the goal, there is uncertainty about when poorer matching is outweighed by the detrimental effects of longer wait time on the deceased-donor list. Should we enter antibodyincompatible donor-recipient pairs into national kidney

This article is part of the topical collection on "What's New in Renal Transplantation?"

Nizam Mamode

nizam.mamode@gstt.nhs.uk

1 Department of Transplantation, Guy's and St Thomas' NHS Foundation Trust, London, England SE1 9RT, UK

2 Renal Offices, 6th Floor Borough Wing, Guy's Hospital, Great Maze Pond, London, England SE1 9RT, UK

3 Department of Paediatric Nephrology, Great Ormond Street Hospital for Children NHS Foundation Trust, Great Ormond Street, London, England WC1N 3JH, UK sharing schemes, and should we include compatible pairs to achieve better matching? Some children will receive an organ from an altruistic living donor, but many will remain on the deceased-donor waiting list. Should we consider donation after cardiac death (DCD; nonbeating heart) and marginal donors for some prospective paediatric renal transplant recipients (pRTR)? What is the role of ex vivo normothermic perfusion for such cases? Should we accept neonatal and infant en bloc donors for prospective pRTR, although it may entail increased thrombotic risk? Which children should receive induction agents for their first transplant when they may receive multiple transplants during their childhood? Should we use monoclonal and polyclonal antibodies more readily in adolescents at risk of losing their renal allografts due to nonadherence? Would monthly infusions of belatacept help such patients adhere to conventional immunosuppression regimes?

Historically, evidence was gleaned from our adult renal transplant colleagues, but we are now performing more studies and clinical trials, including multicentre randomised controlled studies, in pRTR. This is increasing our knowledge and the armamentarium of available immunosuppressive agents in a group of patients who are at increased risk of infectious complications. There is increased Epstein-Barr virus naivety resulting in increased rates of posttransplant lymphoproliferative disorder in our paediatric population. However, some children require increased immunosuppression, such as those undergoing blood group ABO-incompatible and/or HLAincompatible renal transplants and those developing antibody-mediated rejection. Is there a role for mechanistic target of rapamycin (mTOR) inhibitors to promote long-term graft survival, and can newer depleting agents such as bortezomib be used? 
This topical collection in Paediatric Nephrology aims to make it easier for the multidisciplinary team to obtain answers to these questions by drawing together the latest up-to-date basic science and research papers, including reports from clinical trials-as well as clinically relevant reviews on paediatric renal transplantationinto one place. For example: we have upcoming invited reviews on the new generation of $\mathrm{ABO}$ antibody diagnostics, on tissue damage processes and mitochondrial mechanisms in ischaemic reperfusion injury and how proteomics could provide insight into tissue damage and repair processes. It features reviews on preventing delayed graft function, managing failing allografts and transitioning adolescent renal transplant recipients. We also focus on the future of transplantation, state-of-theart immunosuppression protocols, developments in regulatory and stem cell therapies and cutting-edge research in growing organs for transplantation.
We bring this exciting venture to you with the intention of improving outcomes for patients and the dedicated families who tirelessly support them.

Acknowledgements This project was supported by the National Institute for Health Research (NiHR) Biomedical Research Centres based at Guy's and St Thomas' National Health Service (NHS) Foundation Trust and King's College London and the Great Ormond Street Hospital for Children NHS Foundation Trust and University College London. The views expressed are those of the authors and not necessarily those of the NHS, the NiHR or the Department of Health.

\section{Compliance with ethical standards}

Conflicts of interest The authors have no conflicts of interest.

Ethical approval This article does not contain any studies with human or animal subjects performed by the any of the authors. 